\title{
Application of three-dimension information acquisition technology in the badminton Hawkeye system
}

\author{
$\mathrm{Li} \mathrm{Li} i^{1, a}$ \\ ${ }^{1}$ Physical Education Department of Guangxi University, China \\ aili807@foxmail.com
}

Keywords: badminton Hawkeye, binocular stereo vision, 3D information acquisition

\begin{abstract}
The three-dimensional information of the badminton was obtained with epipolar constrain after the cameras of the badminton Hawkeye system were calibrated. The three-dimensional space coordinate of the badminton mass center was got and the flying curve of the badminton was fitted, which have laid a foundation to the 3D construction of the badminton Hawkeye system and the judgment of the placement of the badminton.
\end{abstract}

\section{Introduction}

The three-dimensional information is obtained after the cameras are calibrated. Since the 1990s, the application of the three-dimensional information acquisition technology is got more attention in the competitive sports ${ }^{[1]}$.

The research and practical application of the researchers has made a great contribution to the three dimensional information acquisition technology. The contact measurement method was applied in the early times, which typical applications were three coordinate measuring machine (CMM). Radar method was raised based on radar ranging principle. After that, new aspect of the three-dimensional information were opened by the geometrical optics focusing method, holographic interferometry, Fresnel diffraction technique et al based on the theory of optics and optical information.

The badminton Hawkeye system in this paper is a binocular stereo visual system. The three-dimensional information of the badminton is obtained with epipolar constrain after the cameras of the badminton Hawkeye system is calibrated, which have laid a foundation to the 3D construction of the badminton Hawkeye system and the judgment of the placement of the badminton.

\section{Binocular stereo visual system model}

\section{Epipolar constrain}

The research ${ }^{[6,7]}$ show that epipolar constrain could provide more simple conditions for binocular stereo visual system. The matched point narrow the range to a line of the whole image, which greatly narrow the searching range. Epipolar geometry relationship on the binocular vision system is shown in fig.1. 


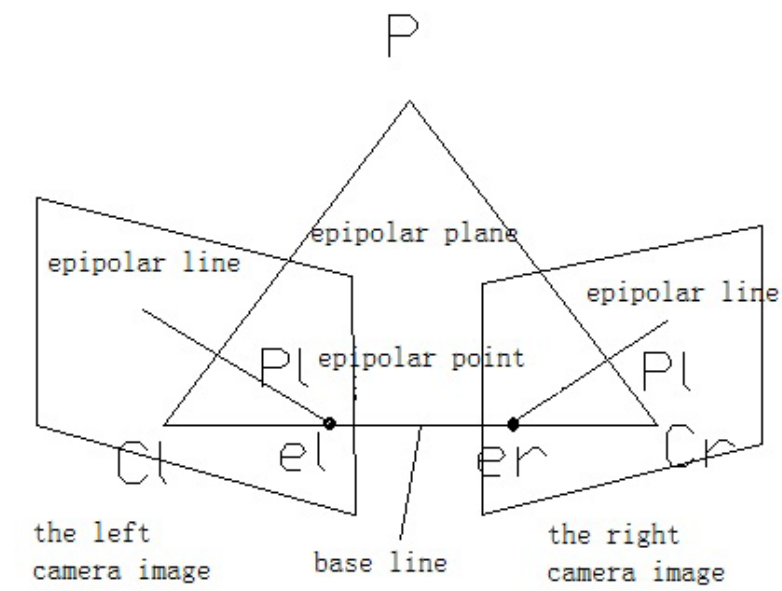

Fig.1 Epipolar geometry relationship on the Binocular Vision System

Where $\mathrm{P}$ is the any point in the space, $\mathrm{P}_{1}$ is the project point in the left camera image, $\mathrm{P}_{\mathrm{r}}$ is the project point in the right camera image; $\mathrm{C}_{1} \mathrm{C}_{\mathrm{r}}$ is the baseline of the connection line of the optic center of the two cameras; epipolar plane $\mathrm{PC}_{1} \mathrm{C}_{\mathrm{r}}$ is the plane determined by $\mathrm{P}$ and the optical center of the two cameras; epipolar line $\mathrm{P}_{1} \mathrm{e}_{1}$ and $\mathrm{P}_{\mathrm{r}} \mathrm{e}_{\mathrm{r}}$ is the intersection line of the epipolar plane and the image plane; epipolar point $e_{1}$ and $e_{r}$ is the intersection point of the baseline and the two image planes of cameras. If the placement of $\mathrm{P}_{1}$ in the right image is known, the corresponding point is certainly in its epipolar line, also the vice versa.

\section{Binocular stereo visual mode in optional position}

The schematic of binocular vision system is shown in fig.2.

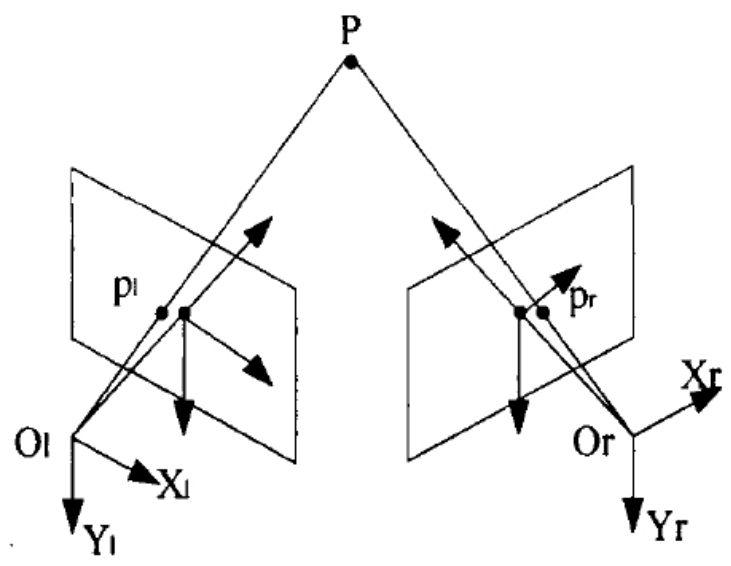

Fig.2 Schematic of Binocular Vision System

For a space point $\mathrm{P}$, the corresponding coordinate in the two cameras are $P_{l}\left(\mathrm{X}_{l}, \mathrm{Y}_{l}\right)$ and $P_{r}\left(\mathrm{X}_{r}, \mathrm{Y}_{r}\right)$. There's following relations:

$$
\left\{\begin{array}{c}
X_{l}=f \frac{x_{w}}{z_{w}} \\
X_{r}=f \frac{\left(\mathrm{x}_{w}-\mathrm{B}\right)}{z_{w}} \\
Y=f \frac{y_{w}}{z_{w}}
\end{array}\right.
$$

The optical parallax $X_{1}-X_{r}$ is obtained from equation (1). $\mathrm{f}$ is the focal length of the camera. So the three-dimensional coordinate of the corresponding feature points in the camera coordinate could express as: 


$$
\left\{\begin{array}{l}
x_{c}=\frac{B \cdot X_{l}}{X_{l}-X_{r}} \\
y_{c}=\frac{B \cdot Y}{X_{l}-X_{r}} \\
z_{c}=\frac{B \cdot f}{X_{l}-X_{r}}
\end{array}\right.
$$

From equation (2), the three-dimensional coordinate of $\mathrm{P}$ is determined only the corresponding point of the right camera image could be found after $\mathrm{P}$ in the left camera is found. $\left(\mathrm{u}_{l}, \mathrm{~V}_{l}\right)$ and $\left(u_{r}, v_{r}\right)$ are defined as the corresponding point in the images; $u_{0}, v_{0}, f_{x}, f_{y}$ are the internal parameter of the camera, so the coordinate could express as:

$$
\left\{\begin{array}{c}
x_{w}=\frac{B\left(\mathrm{u}_{l}-\mathrm{u}_{0}\right)}{u_{l}-u_{r}} \\
y_{w}=\frac{B f\left(v_{l}-v_{0}\right)}{f_{y}\left(u_{l}-u_{r}\right)} \\
z_{w}=\frac{B \cdot f_{x}}{u_{l}-u_{r}}
\end{array}\right.
$$

From equation (3), the three-dimensional space coordinate of $\mathrm{P}$ could be solve if the internal and external parameters are obtained.

The transformation relation from world coordinate $\mathrm{P}\left(x_{w}, y_{w}, z_{w}\right)$ to camera coordinate $\mathrm{p}\left(x_{c}, y_{c}, z_{c}\right)$ could be expressed as:

$$
\left[\begin{array}{l}
x_{c} \\
y_{c} \\
z_{c}
\end{array}\right]=R\left[\begin{array}{l}
x_{w} \\
y_{w} \\
z_{w}
\end{array}\right]+T
$$

Where $\mathrm{R}$ is rotation matrix and $\mathrm{T}$ is translation vector.

\section{Results and analysis}

The three-dimensional space coordinate according the two-dimensional coordinate is computed by HALCON. The original point is set in the top left corner of the badminton court. The right direction is $\mathrm{X}$ axis direction, the backward direction is $\mathrm{Y}$ axis direction, direction of perpendicular to the badminton curt is $\mathrm{Z}$ axis direction.

The original data of the 3D information of spatial points is shown in table 1.

Table1 3Dinformation of spatial points

\begin{tabular}{c|c|c|c}
\hline \multirow{2}{*}{} & \multicolumn{3}{|c}{ Coordinate value $(\mathrm{mm})$} \\
\cline { 2 - 4 } & $\mathrm{X}$ & $\mathrm{Y}$ & $\mathrm{Z}$ \\
\hline 1 & 445.472 & 859.209 & 981.597 \\
\hline 2 & 441.282 & 817.896 & 974.599 \\
\hline 3 & 428.720 & 688.610 & 924.504 \\
\hline 4 & 423.848 & 633.013 & 893.723 \\
\hline 5 & 419.789 & 578.340 & 852.489 \\
\hline 6 & 417.341 & 524.311 & 800.113 \\
\hline 7 & 407.154 & 410.650 & 667.477 \\
\hline 8 & 396.365 & 301.894 & 467.121 \\
\hline
\end{tabular}




\begin{tabular}{c|c|c|c}
\hline 9 & 387.522 & 191.786 & 182.080 \\
\hline 10 & 380.908 & 126.689 & 100.168 \\
\hline 11 & 370.601 & 76.057 & 169.373 \\
\hline
\end{tabular}

We can see from table 1 that the tenth point is the lowest point of $\mathrm{Z}$ value. The badminton bounced after landing (the $11^{\text {th }}$ point). The curve based on the original data is shown in fig.3.

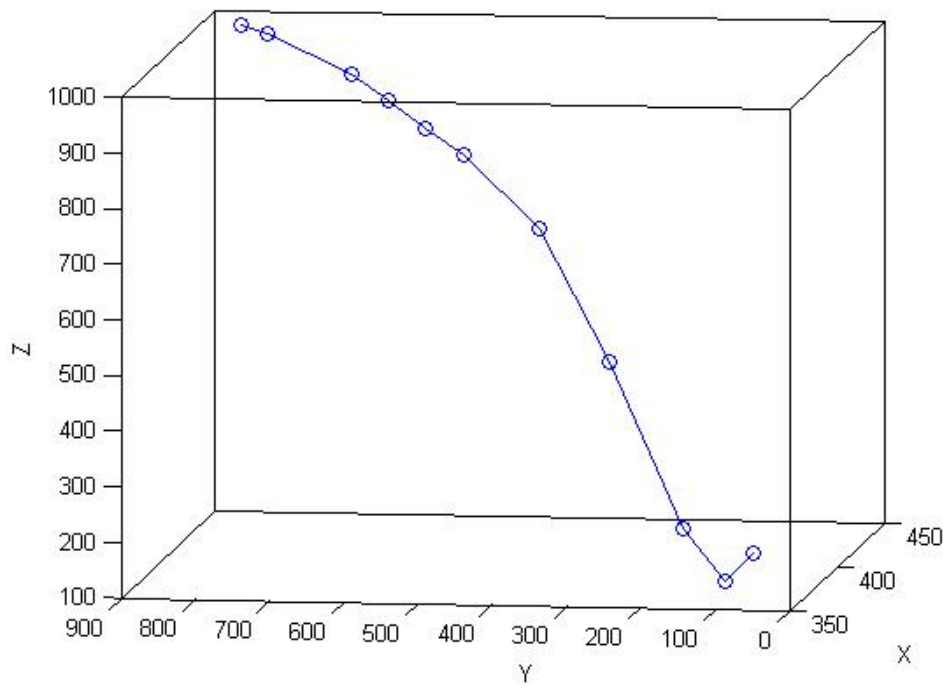

Fig.3 The curve based on the original data

We can see from fig. 3 that the curve based on the original data is not smooth, which is not consistent with the badminton flying orbit. What's more, the minimum $\mathrm{Z}$ value is too large, that the badminton is still not landing. So parabola fitting is needed.

The least square principle is used to fit the curve base on the requirement of the three-dimensional reconstruction needs a certain amount of data. 50 data is fitted, and counted the data of first drop of the badminton, there's 51data in total.

Table2 3D information of spatial points after curve fitting

\begin{tabular}{c|c|c|c}
\hline \multirow{2}{*}{ number } & \multicolumn{3}{|c}{ Coordinate value (mm) } \\
\cline { 2 - 4 } & $\mathrm{X}$ & $\mathrm{Y}$ & $\mathrm{Z}$ \\
\hline 1 & 450.429 & 924.625 & 1044.658 \\
\hline 2 & 448.976 & 908.334 & 1031.798 \\
\hline$\ldots$ & $\ldots$ & $\ldots$ & $\ldots$ \\
\hline 47 & 384.284 & 159.237 & 120.606 \\
\hline 48 & 382.865 & 142.988 & 98.238 \\
\hline 49 & 381.472 & 126.684 & 67.352 \\
\hline 50 & 380.908 & 119.389 & 50.000 \\
\hline 51 & 370.601 & 76.057 & 69.373 \\
\hline
\end{tabular}




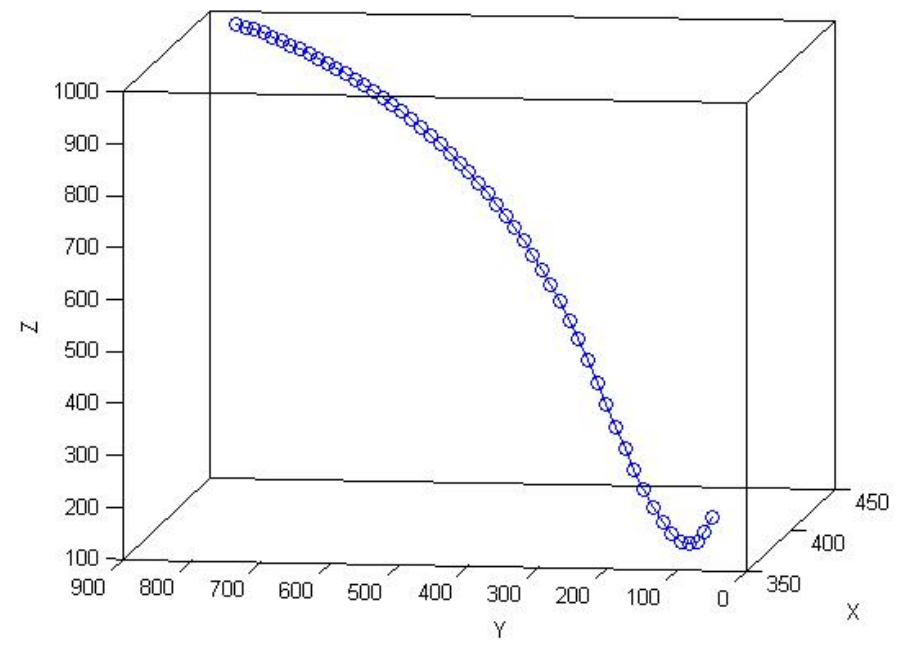

Fig.4 The curve based on the data after curve fitting

From fig.2, the fiftieth point is the lowest one in $\mathrm{Z}$ direction, which $\mathrm{Z}=50.000$. The point which $Z=50$ won't be fitted if the $Z$ value of the original data is less than 50 , but fitting the point that $Z=50$ on the contrary. The curve is more smoothness after fitting by least square principle, and it also eliminate the error, which makes the data more accuracy and reasonable.

\section{References}

[1] Huo Fang. The application of instant reviewing system in tennis match. Bulletin of Sport Science \& Technology. 2007,15(9):10-11

[2] R. A. Jarvis. A perspective on Range Finding Techniques for Computers for Computer Vision, IEEE Trans. On PA\&MI, March, 1983,Vol. 5(2),p122-139

[3] Frank Chen, Cordon M. Brown, Mumin Song. Overview of three-dimensional shape measurement using optical methods. Optical Engineering, 2000.1, Vol. 39(1), 10-22

[4] Carsten Steger, Makus Ulrich, Christian Wiedmann. Machine Vision Algorithms and Application [M], Wieley-VCH, 2007.

[5] Dana H. Ballard, Christopher M. Brown. Computer Vison. New Jersey: Prentice-Hall Inc., 1982

[6] Zhou Ji, Shi Jiaoying. A robust algorithm for feature point matching. Computer \& Graphics,2002,26:429-436

[7] Xiang DN, Deng WY, Yan BX. Image token correspondence with constraint of epipolar line. Journal of Beijing Institute of Machinery.2002，17 (4): 21-25

[8] Qin XW, Wen ZF, Qiao WW. Image processing based on OpenCV. Electronic Test.2011,7:39-41

[9] Rafael C. Gonzalez, Richard E. Woods, Steven L. Eddins. Digital Image Processing Using Matlab[M], Prentice Hall, Upper Saddle River, NJ.

[10] McKenna Setal, tracking groups of people. Computer Vision and Image Understanding, 2000,80(1):42-56 